\title{
Spinal Anesthesia Performed Under Previous Ultrasound Examination Guidance. Report of 30 Cases Scheduled for Orthopedic Surgery
}

\begin{abstract}
Introduction: During the last ten years, ultrasound (US) guidance has revolutionized regional anesthesia field in many countries. Despite this, its role in central neuroaxial blockade has been underappreciated by anesthesiologists. Ultrasound examination is useful to identify and mark intervertebral levels, needle insertion point for a midline approach and also to estimate needle orientation and depth from skin surface to subarachnoid space. This is especially helpful in difficult cases. In addition, ultrasound identification of the intervertebral levels is more accurate than clinical estimation using the intercristal line. It also increases the success rate of dural puncture on the first needle insertion attempt. In this article, we share our experience in 30 consecutive central neuroaxial blockades guided by previous ultrasound examination.
\end{abstract}

Methods: Thirty consecutive patients scheduled for orthopedic surgery under spinal anesthesia were included in this prospective analysis. Patients were placed in a sitting position. Ultrasound examination for skin markings and measurements was performed. After this was completed, anesthesia residents performed spinal anesthesia following those marks. Data required for analysis was recorded by an independent observer.

Results: Mean time of ultrasound examination before spinal block performing was 5.6 minutes. Success rate of dural puncture on the first needle insertion attempt was $75 \%$. In the rest $25 \%$, the median number of needle passes required for success was $1 .{ }^{1-3}$ It is important to state that $10 \%$ of the patients had previous spine surgery, $10 \%$ presented scoliosis with a severe deformity and $20 \%$ had BMI of 32 or more.

Conclusion: Ultrasound-guided neuraxial blockade is a useful technique, especially for difficult patients. It is associated with a high success rate of dural puncture on the first needle insertion attempt and a reduction in the median number of needle passes required for success, even when spinal anesthesia was performed by in-training physicians.

Keywords: Regional anesthesia, Ultrasound, Neuroaxial blockade
Volume I Issue 5 - 2014

\author{
Guardabassi Diego Sebastian, Perrin Turenne \\ Hugo, Dominguez Garcia Maria Ximena, \\ Vescovo Anibal \\ Department of Anesthesia, Hospital Italiano de Buenos Aires, \\ Argentina
}

Correspondence: Guardabassi Diego Sebastian, Department of Anesthesia, Hospital Italiano de Buenos Aires, Pte J D Peron 4190 - I I floor, Buenos Aires, Argentina, Tel 54-I I-63375398, Email guardabassidiego@hotmail.com

Received: November 03, 2014 | Published: November 18 2014

\section{Introduction}

During the last ten years, ultrasound (US) guidance has revolutionized regional anesthesia field in Argentina and many countries, particularly peripheral nerve blockade. Despite this affirmation, its role in central neuroaxial blockade has been underappreciated by anesthesiologists, partly because of the relative efficacy of the landmark-guided technique and partly because of the perceived difficulty in imaging through the narrow acoustic windows produced by the bony framework of the spine. ${ }^{1,2}$

However, this is also the basis for the utility of ultrasound in neuraxial blockade: if an interlaminar window that permits passage of sound waves into the vertebral canal can be identified, the same window will permit passage of a needle into the epidural or intrathecal space. In addition, it is useful to identify and mark intervertebral levels, needle insertion point for a midline approach and also to estimate needle orientation and depth from skin surface to subarachnoid space. ${ }^{2,3}$

By following this ultrasound approach, it is possible to perform the spinal or epidural anesthetic in the usual fashion, guided by the skin markings and depth measurements. This is especially helpful in difficult cases ${ }^{4}$ like: morbid obesity, ${ }^{5}$ which makes palpation of bony surfaces impossible; spine deformity such as previous spine surgery or scoliosis ${ }^{6,7}$ where performing a neuroaxial block technique is traumatic and often requires multiple punctures; trauma patients, ${ }^{8}$ when patient mobilization is difficult and does not allow to find a correct positioning before performing a central neuroaxial blockade.

In addition to this, ultrasound can identify the intervertebral levels by counting spinous processes or laminae upward from the sacrum; this method is more accurate than clinical estimation using the intercristal line $(67-90 \%$ vs. $35-50 \%)$. It also increases the success rate of dural puncture on the first needle insertion attempt and reduces the median number of needle passes required for success, as it was demonstrated in several previous publications. ${ }^{1,2,9}$ In this article, we share our experience in 30 consecutive central neuroaxial blockades guided by skin markings and measurements made by ultrasound.

\section{Methods}

The study was performed at Hospital Italiano de Buenos Aires, Argentina. After obtaining institutional review board approval. 30 consecutive patients scheduled for orthopedic surgery under spinal anesthesia were included in this prospective analysis.

\section{Technique of anesthesia}

Routine monitors (pulse oximeter, noninvasive blood pressure cuff, and electrocardiogram) were applied, and intravenous access 
was established. Patients were placed in a sitting position, with the head and trunk bended slightly forward. Ultrasound examination for skin markings and measurements was performed then, using a $3,5 \mathrm{MHz}$ curve array US probe (Sonosite Nerve).

Each examination was done only by previously trained staff anesthesiologists (50 ultrasound spine examinations), by following the next steps:

Firstly, a transverse ultrasound scan was done in order to identify and mark midline. Spinous processes could be seen in this view (Figure 1). Secondly, a paramedian sagittal ultrasound scan was performed to identify and mark each intervertebral level, by counting spinous processes or laminae upward from the sacrum. A corresponding skin mark was made at the midpoint of the probe's long edge (Figures $2 \&$ $3)$.

Thirdly, a transverse ultrasound scan was made at the intervertebral level which was appropriate for needle placement and proper identification and markings of interspinous space were established (Figure 4). The midline was centered on the ultrasound screen, and skin marks were made at the midpoint of the probe's long and short edges. The intersection of these two marks provided an appropriate needle insertion point for a midline approach to the intrathecal space at that level. Distance from skin to subarachnoid space was measured. Cephalic or caudal tilt of US probe when getting the best plane image was considered for needle orientation during block performance. This process was repeated at one intervertebral level above the one considered for spinal block attempt.

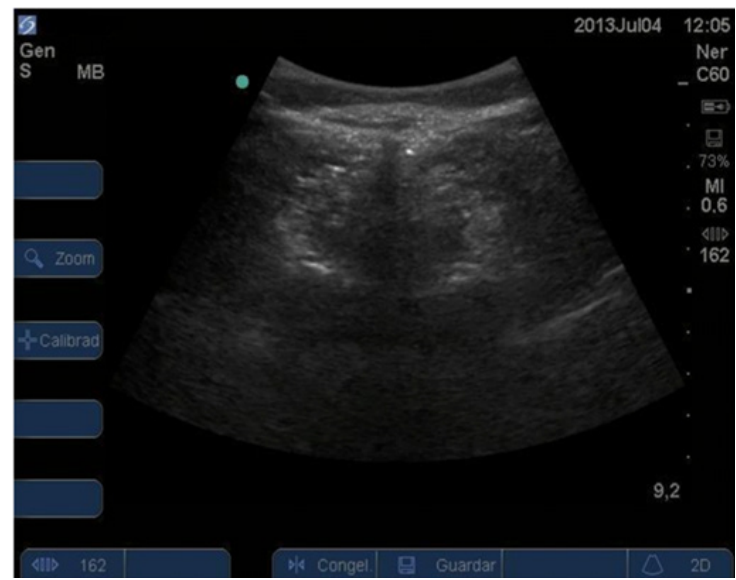

Figure I Transverse ultrasound scan was done in order to identify and mark midline. Spinous processes (SP) could be seen in this view.

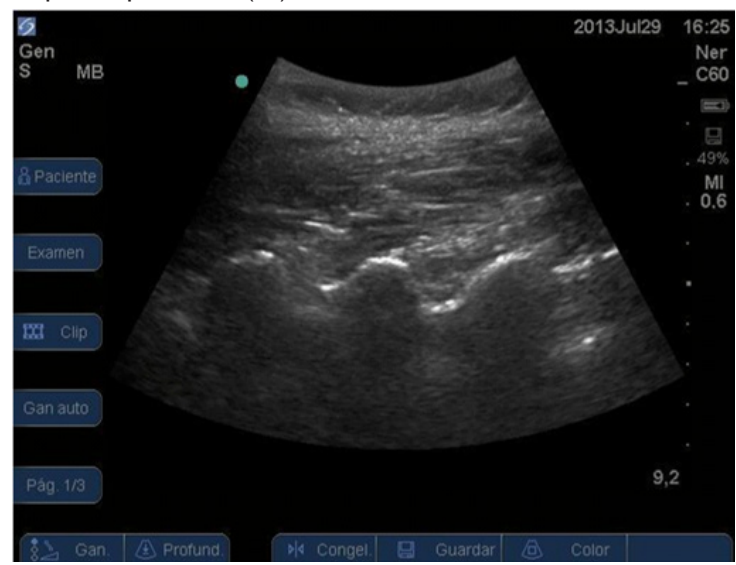

Figure 2 Paramedian sagittal ultrasound scan was performed to identify and mark each intervertebral level, by counting laminae $(L)$ upward from the sacrum.

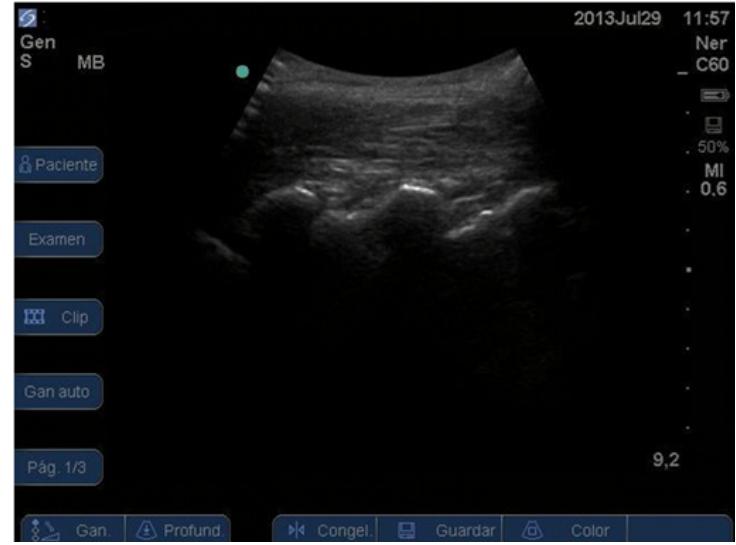

Figure 3 Paramedian sagittal scan. Intervertebral level determination, by counting laminae $(\mathrm{L})$ upward from the sacrum(S).

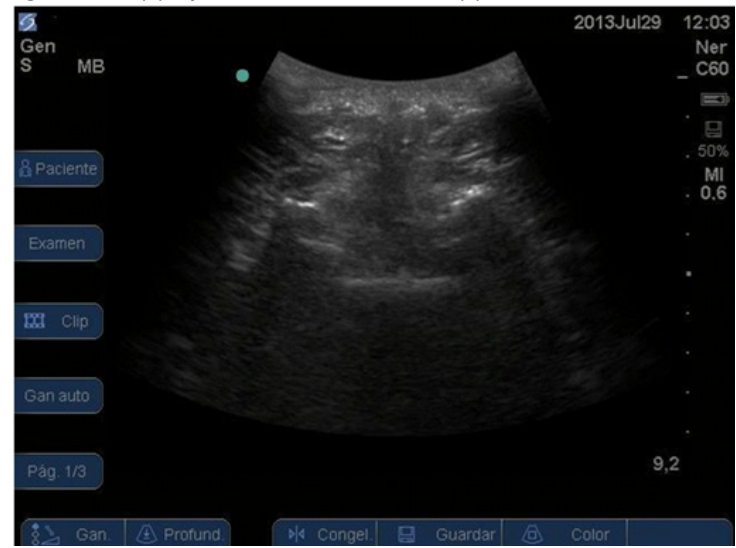

Figure 4 Transverse ultrasound scan was made at the appropriate intervertebral level. Posterior complex (PC), dura and flavum ligament, and anterior complex $(\mathrm{AC})$, posterior longitudinal ligament, could be seen in this view.

Finally, a new brief ultrasound examination was done in order to check proper skin marks. If any error was found, the whole process was repeated. Time employed to perform full examination was recorded by an independent observer for posterior analysis. After ultrasound examination was completed, anesthesia residents proceeded to perform spinal anesthesia. Under sterile conditions, a 9-cm 26G pencil point spinal needle (Whitacre) was inserted following skin marks at the chosen intervertebral level and with the orientation determined in previous US exam by the staff anesthesiologist. Previous infiltration of skin at the site of puncture with lidocaine $1 \%(3 \mathrm{~mL})$ was done, without palpation of bony structures. Needle was continuously advanced until reaching subarachnoid space. In case of bone contact during needle advancement, it was reoriented and this process was repeated. Each reorientation was considered as a needle pass. In the case that, after 5 needle passes, it was not possible to reach subarachnoid space, a new puncture was made at a different intervertebral level.

An independent observer recorded all these data: time to perform full US examination; number of repeated US examinations due to skin marks errors; needle insertion attempts required for success; changes of intervertebral space due to technical failure.

\section{Results}

Between June-August 2013, 30 patients undergoing scheduled orthopedic surgery under spinal anesthesia were analyzed. Demographic profile is represented in Table 1. It is important to state 
that $10 \%$ of the patients had previous spine surgery, $10 \%$ presented scoliosis with a severe deformity and $20 \%$ had BMI of 32 or more. In all cases, it was possible to perform the spinal anesthesia in the usual fashion, guided by the skin markings and depth measurements made by ultrasound. Mean time spent for ultrasound examination before spinal block performing was 5.6 minutes. Skin marking correction was needed in only one patient. Success rate of dural puncture on the first needle insertion attempt was $75 \%$. In the rest $25 \%$, the median number of needle passes required for success was 1 (range 1-3). No puncture was performed at an intervertebral space different of the one chosen by previous examination. A summary of these results could be visualized in Table 2.

Table I Patients Characteristics

\begin{tabular}{ll}
\hline Age (mean) & 56 years \\
\hline Sex (F/M) & $6 / 14$ \\
Weight (mean) & $73,4 \mathrm{~kg}$ \\
Height (mean) & $165 \mathrm{~cm}$ \\
BMI>32 (x/x-\%) & $3 / 20(15 \%)$ \\
Previous spine surgery & $2 / 20(10 \%)$ \\
Scoliosis & $2 / 20(10 \%)$ \\
Trauma surgery & $1 / 10(5 \%)$ \\
\hline
\end{tabular}

\section{Table 2 Results}

\begin{tabular}{ll}
\hline $\begin{array}{l}\text { Success rate of dural puncture on the first needle } \\
\text { insertion attempt }\end{array}$ & $75 \%$ \\
\hline $\begin{array}{l}\text { Number of needle passes, median (range) } \\
\text { Mean time spent for ultrasound examination (min), }\end{array},_{5,6}(I-3)$ \\
mean (SD)
\end{tabular}

\section{Discussion}

Several previous studies have demonstrated the accuracy of ultrasound guidance for skin markings and measurements before performing a central Neuroaxial blockade. ${ }^{1-4,6}$ Chin et al. ${ }^{9}$ reported, in 120 patients undergoing total knee replacement under spinal anesthesia, a success rate of dural puncture on the first needle insertion attempt of $62 \%$ when performing blockade with ultrasound guidance compared with $32 \%$ of success when classic palpation technique was done. This article also showed a reduction of three in the median number of needle passes required for success. Grau et al. ${ }^{3,4}$ has found similar results in many publications. All these data were similar with the results we found in the group studied. Ultrasound guidance was demonstrated to be quite useful for skin markings in difficult patients in a number of studies. ${ }^{4-10}$ It is important to state that $35 \%$ of the patients we analyzed could be included in this subgroup. We consider this could highlight the success rate on the first needle insertion attempt achieved in this population.

Ultrasound can identify the intervertebral levels by counting spinous processes or laminae upward from the sacrum; this method is more accurate than clinical estimation using the intercristal line. ${ }^{11}$ Correlation between these two methods occurs only in 35$55 \%$ of the cases. ${ }^{12,13}$ Compared with other imaging modalities, such as magnetic resonance imaging, computed tomography and plain radiographs of the lumbar spine, ultrasound accurately identified a spinous process or intervertebral space $68-76 \%$ of the time. It is worth noting that any inaccuracy observed with ultrasound is likely to be within one interspace of the true level, rather than two or three interspaces, as may occur with palpation of surface landmarks. . $^{14,12}$

Errors are also more likely in the early stages of learning to perform ultrasonography of the spine and accuracy rates of $90 \%$ or greater probably can be achieved with adequate training and experience. Mistakes usually result from misidentification of the L5-S1 junction or failure to recognize developmental anomalies of the lumbosacral junction, which occur in approximately $12 \%$ of the general population. ${ }^{15-17}$ We were able to perform with no difficulties the ultrasound examination in every patient included. However, correct identification of the intervertebral level was not compared with another superior imaging technique. An interesting topic mentioned in many studies was the usefulness of this method as a tool for new specialists and residents' education and the highly successful rate that was obtained. ${ }^{18}$ In this study, $100 \%$ of the spinal blockades were performed by anesthesia residents, and similar results with previous published studies were reached.

Finally, it is worth noting that ultrasound scanning demands a short period of time, about 5 to 6 minutes. ${ }^{19}$ There is no study that compares the time expended to perform a spinal blockade with and without previous ultrasound marking.

\section{Conclusion}

Ultrasound-guided neuraxial blockade is a useful technique that can, among other things, help practitioners more accurately identify intervertebral levels, estimate depth to the subarachnoid space, and locate an appropriate interlaminar space for needle insertion. The utility of the ultrasound-guided approach is most evident in patients in whom technical difficulty is expected because of poor surface anatomic landmarks (e.g., in obesity or after spinal surgery) or distorted spinal anatomy (e.g., scoliosis). It is associated with a high success rate of dural puncture on the first needle insertion attempt and a reduction in the median number of needle passes required for success. It is an excellent tool for resident education and practice. Time of proper scanning is usually short and could be improved with adequate training and more clinical experience. Randomized controlled trials are necessary to compare the results shown in our population.

\section{Funding details}

None.

\section{Acknowledgments}

None.

\section{Conflicts of interest}

Authors declare that there is no conflicts of interest.

\section{References}

1. Chin KJ, Perlas A. Ultrasonography of the lumbar spine for neuraxial and lumbar plexus blocks. Curr Opin Anaesthesiol. 2011;24(5):567-572.

2. Chin KJ, Karmakar MK, Peng P. Ultrasonography of the adult thoracic and lumbar spine for central neuraxial blockade. Anesthesiology. 2011;114(6):1459-1485.

3. Grau T, Leipold RW, Conradi R, et al. Efficacy of ultrasound imaging in obstetric epidural anesthesia. J Clin Anesth. 2002;14:169-175.

4. Grau T, Leipold RW, Conradi R, et al. Ultrasound control for presumed difficult epidural puncture. Acta Anaesthesiol Scand. 2001;45(6):766-771.

5. O’Donnell D, Prasad A, Perlas A. Ultrasound-assisted spinal anesthesia in obese patients. Can J Anaesth. 2008;56(12):982-983.

6. Chin KJ, Macfarlane AJ, Chan V, et al. The use of ultrasound to facilitate spinal anesthesia in a patient with previous lumbar laminectomy and fusion:a case report. J Clin Ultrasound. 2009;37(8):482-485. 
7. McLeod A, Roche A, Fennelly M. Case series:Ultrasonography may assist epidural insertion in scoliosis patients. Can $J$ Anaesth. 2005;52(7):717-720.

8. Chin KJ, Chan VW, Ramlogan R, et al. Real-time ultrasound-guided spinal anesthesia in patients with a challenging spinal anatomy:Two case reports. Acta Anaesthesiol Scand. 2010;54(2):252-255.

9. Chin KJ, Perlas A, Chan V, et al. Ultrasound imaging facilitates spinal anesthesia in adults with difficult surface anatomical landmarks. Anesthesiology. 2011;115(1):94-101.

10. Peng PW, Rofaeel A. Using ultrasound in a case of difficult epidural needle placement. Can J Anaesth. 2006;53(3):325-326.

11. Whitty R, Moore M, Macarthur A. Identification of the lumbar interspinous spaces:Palpation versus ultrasound. Anesth Analg. 2008;106(2):538-540.

12. Furness G, Reilly MP, Kuchi S. An evaluation of ultrasound imaging for identification of lumbar intervertebral level. Anaesthesia. 2002;57(3):277-280.

13. Schlotterbeck H, Schaeffer R, Dow WA, et al. Ultrasonographic control of the puncture level for lumbar neuraxial block in obstetric anaesthesia. Br J Anaesth. 2008;100(2):230-234.
14. Halpern SH, Banerjee A, Stocche R, et al. The use of ultrasound for lumbar spinous process identification: A pilot study. Can J Anaesth. 2010;57(9):817-822.

15. Bron JL, van Royen BJ, Wuisman PI. The clinical significance of lumbosacral transitional anomalies. Acta Orthop Belg. 2007;73:687-695.

16. Scapinelli R. Morphological and functional changes of the lumbar spinous processes in the elderly. Surg Radiol Anat. 1989;11(20):129-133.

17. Whitty RJ, Maxwell CV, Carvalho JC. Complications of neuraxial anesthesia in an extreme morbidly obese patient for Cesarean section. Int J Obstet Anesth. 2007;16(2):139-144.

18. Vallejo MC, Phelps AL, Singh S, et al. Ultrasound decreases the failed labor epidural rate in resident trainees. Int J Obstet Anesth. 2010; 19(4):373-378.

19. Margarido CB, Arzola C, Balki M, et al. Anesthesiologists' learning curves for ultrasound assessment of the lumbar spine. Can J Anaesth. 2010;57(2):120-126. 